\title{
BALANCE GENERAL Y ESTADO DE GANANCIAS Y PÉRDIDAS: FUENTE DE INFORMACIÓN MEDIOAMBIENTAL
}

Dr. Ernesto Augusto Polar Falcón*

\begin{abstract}
RESUMEN
La contabilidad financiera, llamada también contabilidad tradicional, tiene como objetivo primordial la preparación de los estados financieros, formados por los informes principales y las notas a los estados financieros. Los dos informes principales básicos denominados Balance General y Estado de Ganancias y Pérdidas contienen rubros con partidas medioambientales, que es necesario ubicar para otorgar información relacionada con la contabilidad medioambiental.

La ubicación de esta información medioambiental es el objetivo de este artículo preparado especialmente para la revista Quipukamayoc que edita el Instituto de Investigación de Ciencias Financieras y Contables de la Facultad de Ciencias Contables de la Universidad Nacional Mayor de San Marcos.

Para alcanzar el objetivo propuesto ha sido necesario seguir investigaciones de acuerdo al programa de trabajo preparado en el Estudio de Investigación 2004 del autor del presente artículo, cuyo título es: «La Importancia de la Contabilidad Medioambiental en el Marco de la Información Financiera Empresarial».

La contabilidad tradicional contiene información referida a activos y pasivos medioambientales, ubicados en el Balance General; así como ingresos, costos y gastos medioambientales, que forman parte de la información económica sustentada en el Estado de Ganancias y Pérdidas.

En el presente artículo estas partidas con incidencia medioambiental en el Balance General y en el Estado de Ganancias y Pérdidas se presentan como una primera etapa de trabajo de investigación analítica; como una segunda etapa se requiere realizar un estudio de mayor profundidad para precisar las procedencias de estas partidas medioambientales y buscar las soluciones para disminuirlas, modificarlas o anularlas en preservación del medio ambiente.

También se debe informar sobre las partidas contingentes medioambientales aplicando los criterios y recomendaciones que da la NIC 37- «Provisiones, Activos Contingentes y Pasivos Contingentes». Estas partidas contingentes medioambientales pueden presentarse en las Notas a los Estados Financieros en la Memoria de la empresa, en un Informe de Gestión o incorporarse al Informe de Auditoría Financiera.
\end{abstract}

* Docente Principal de la Facultad de Ciencias Contables.

Director del Instituto de Investigación de la Facultad de Ciencias Financieras y Contables.

Miembro del Comité Ejecutivo del Consejo Superior de Investigaciones - UNMSM, Representante del Área Económico-Empresarial. 
1. BALANCE GENERAL CON INFORMACIÓN PORCENTUAL

Al 31 de diciembre del 2003

(Expresado en miles de nuevos soles)

\section{ANEXO N. ${ }^{\circ} 1$}

Se presenta el Balance General de la empresa Algodonera Perú S.A., al 31 de diciembre del 2003, con información financiera porcentual comparativa 2003 -2002 , en miles de nuevos soles.

Tomando como base lo expresado por Rivero Torre, autor del libro Análisis de Balances y Estados Complementarios, cuando afirma que el Balance General debe informar no sólo la situación actual estricta del patrimonio, sino también los demás aspectos complementarios, que ayudan a obtener una visión total de las circunstancias en que está inmerso y que pueden alterarlo en el futuro, tendría cabida la ubicación de partidas medioambientales que otorgarían una información importante para la dirección de la empresa.

\section{ACTIVO-UBICACIÓN DE RUBROS CON PARTIDAS MEDIOAMBIEN-} TALES

Al 31 de diciembre del 2003

(Expresados en miles de nuevos soles)

\section{SUBANEXO 1.1}

De acuerdo con lo expresado en el Activo del Balance General mostrado en el ANEXO N. ${ }^{\circ} 1$ se han ubicado los siguientes tres rubros con información medioambiental, cuyo análisis se presenta en el SUBANEXO 1.1, destacándose los montos y los porcentajes en función al $100 \%$ del total del activo:

\begin{tabular}{|c|c|c|c|c|}
\cline { 2 - 5 } \multicolumn{1}{c|}{} & \multicolumn{4}{c|}{$\begin{array}{c}\text { Con Incidencia } \\
\text { Medioam biental }\end{array}$} \\
\cline { 2 - 5 } & \multicolumn{2}{c|}{$\mathbf{2 0 0 3}$} & \multicolumn{2}{c|}{$\mathbf{2 0 0 2}$} \\
\cline { 2 - 5 } & S/. & $\%$ & S/. & $\%$ \\
\hline - Existencias & 14,525 & 11.0 & 10,396 & 10.1 \\
\hline $\begin{array}{c}\text { - Inm uebles, } \\
\text { Maquinaria } \\
\text { y Equipo }\end{array}$ & 44,612 & 33.8 & 31,927 & 30.9 \\
\hline $\begin{array}{c}\text { - Activos } \\
\text { Intangibles }\end{array}$ & 723 & 0.6 & 472 & 0.5 \\
\hline
\end{tabular}

Comentario: Es importante observar en estos rubros la elevada inversión efectuada en partidas con incidencia medioambiental.

\section{PASIVO-UBICACIÓN DE RUBROS CON PARTIDAS MEDIOAMBIENTALES}

Al 31 de Diciembre del 2003

(Expresados en miles de Nuevos Soles)

\section{SUBANEXO 1.2}

Asimismo, en el Pasivo del Balance General mostrado en el ANEXO N. ${ }^{\circ} 1$ se han ubicado los siguientes dos rubros con información medioambiental, cuyo análisis se presenta en el SUBANEXO N. ${ }^{\circ} 1.2$, destacándose los montos y los porcentajes en función al $100 \%$ del total del pasivo y patrimonio:

\begin{tabular}{|c|c|c|c|c|}
\cline { 2 - 5 } \multicolumn{1}{c|}{} & \multicolumn{3}{c|}{$\begin{array}{c}\text { Con Incidencia } \\
\text { Medioambiental }\end{array}$} \\
\cline { 2 - 5 } \multicolumn{1}{c|}{} & \multicolumn{2}{c|}{2003} & \multicolumn{2}{c|}{2002} \\
\cline { 2 - 5 } \multicolumn{1}{c|}{ S/. } & $\%$ & S/. & $\%$ \\
\hline $\begin{array}{c}\text { - Cuentas por } \\
\text { Pagar } \\
\text { Comerciales }\end{array}$ & 13,322 & 10.1 & 8,409 & 8.1 \\
\hline $\begin{array}{c}\text { - Deudas a } \\
\text { Largo Plazo }\end{array}$ & 8,490 & 6.4 & 4,052 & 4.0 \\
\hline
\end{tabular}

Comentario: En el análisis realizado en estos rubros se han ubicado deudas provenientes de compras de existencias y activos fijos que tienen incidencia medioambiental. 
ALGODONERA PERÚ S.A.

BALANCE GENERAL CON INFORMACIÓN PORCENTUAL

Al 31 de diciembre del 2003

(Expresado en miles de nuevos soles)

\begin{tabular}{|c|c|c|c|c|}
\hline & \multicolumn{2}{|c|}{ ANO 2003} & \multicolumn{2}{|c|}{ ANO 2002} \\
\hline & S/. & $\%$ & S/. & $\%$ \\
\hline \multicolumn{5}{|l|}{ ACTIVO CORRIENTE } \\
\hline Caja y Bancos & 7,060 & 5.3 & 6,354 & 6.2 \\
\hline Cuentas por cobrar comerciales & 25,224 & 19.1 & 22,702 & 22.0 \\
\hline Otras cuentas por cobrar & 7,354 & 5.6 & 5,837 & 5.6 \\
\hline Existencias & 18,163 & 13.7 & 16,346 & 15.8 \\
\hline Gastos pagados por anticipado & $\underline{3,446}$ & 2.6 & $\underline{2,412}$ & 2.3 \\
\hline Total del activo corriente & 61,247 & 46.3 & 53,651 & 51.9 \\
\hline Inversiones permanentes & 132 & 0.1 & 92 & 0.1 \\
\hline Inmuebles, maquinaria y equipo (Neto de & & & & \\
\hline depreciación acumulada) & 68,585 & 51.9 & 48,010 & 46.5 \\
\hline Activos intangibles & 982 & 0.8 & 688 & 0.7 \\
\hline Impuesto a la renta diferido & $\underline{1.204}$ & 0.9 & 843 & 0.8 \\
\hline TOTAL ACTIVO & $\underline{132,150}$ & $\underline{100.0}$ & $\underline{103,284}$ & $\underline{100.0}$ \\
\hline \multicolumn{5}{|l|}{ PASIVO Y PATRIMONIO } \\
\hline \multicolumn{5}{|l|}{ PASIVO CORRIENTE } \\
\hline Cuentas por pagar comerciales & 18,571 & 14.1 & 12,999 & 12.6 \\
\hline Otras cuentas por pagar & 39,255 & 29.7 & 27,479 & 26.6 \\
\hline \multirow{2}{*}{\multicolumn{5}{|c|}{$\begin{array}{l}\text { Porción corriente de las deudas a largo } \\
\text { plazo }\end{array}$}} \\
\hline & 300 & 0.2 & 210 & 0.2 \\
\hline Total del pasivo corriente & 58,126 & 44.0 & 40,688 & 39.4 \\
\hline Deudas a largo plazo & $\underline{14,860}$ & $\underline{11.2}$ & $\underline{10,402}$ & $\underline{10.1}$ \\
\hline TOTAL PASIVO & 72,986 & $\underline{55.2}$ & $\underline{51,090}$ & $\underline{49.5}$ \\
\hline GANANCIAS DIFERIDAS & $\underline{5,271}$ & 4.0 & $\underline{3,690}$ & 3.6 \\
\hline \multicolumn{5}{|l|}{ PATRIMONIO NETO } \\
\hline Capital & 36,895 & 27.9 & 33,205 & 32.1 \\
\hline Reserva Legal & 5,823 & 4.4 & 5,241 & 5.1 \\
\hline Resultados Acumulados & $\underline{11,175}$ & 8.5 & $\underline{10,058}$ & 9.7 \\
\hline TOTAL PATRIMONIO NETO & $\underline{53,893}$ & $\underline{40.8}$ & $\underline{48,504}$ & $\underline{46.9}$ \\
\hline TOTAL PASIVO Y PATRIMONIO NETO & $\underline{132,150}$ & $\underline{100.0}$ & 103,284 & $\underline{100.0}$ \\
\hline
\end{tabular}


SUBANEXO N. ${ }^{\circ} 1.1$

\section{ALGODONERA PERÚ S.A. \\ Al 31 de diciembre del 2003 \\ (Expresado en miles de nuevos soles)}

ACTIVO- UBICACIÓN DE RUBROS CON PARTIDAS MEDIOAMBIENTALES

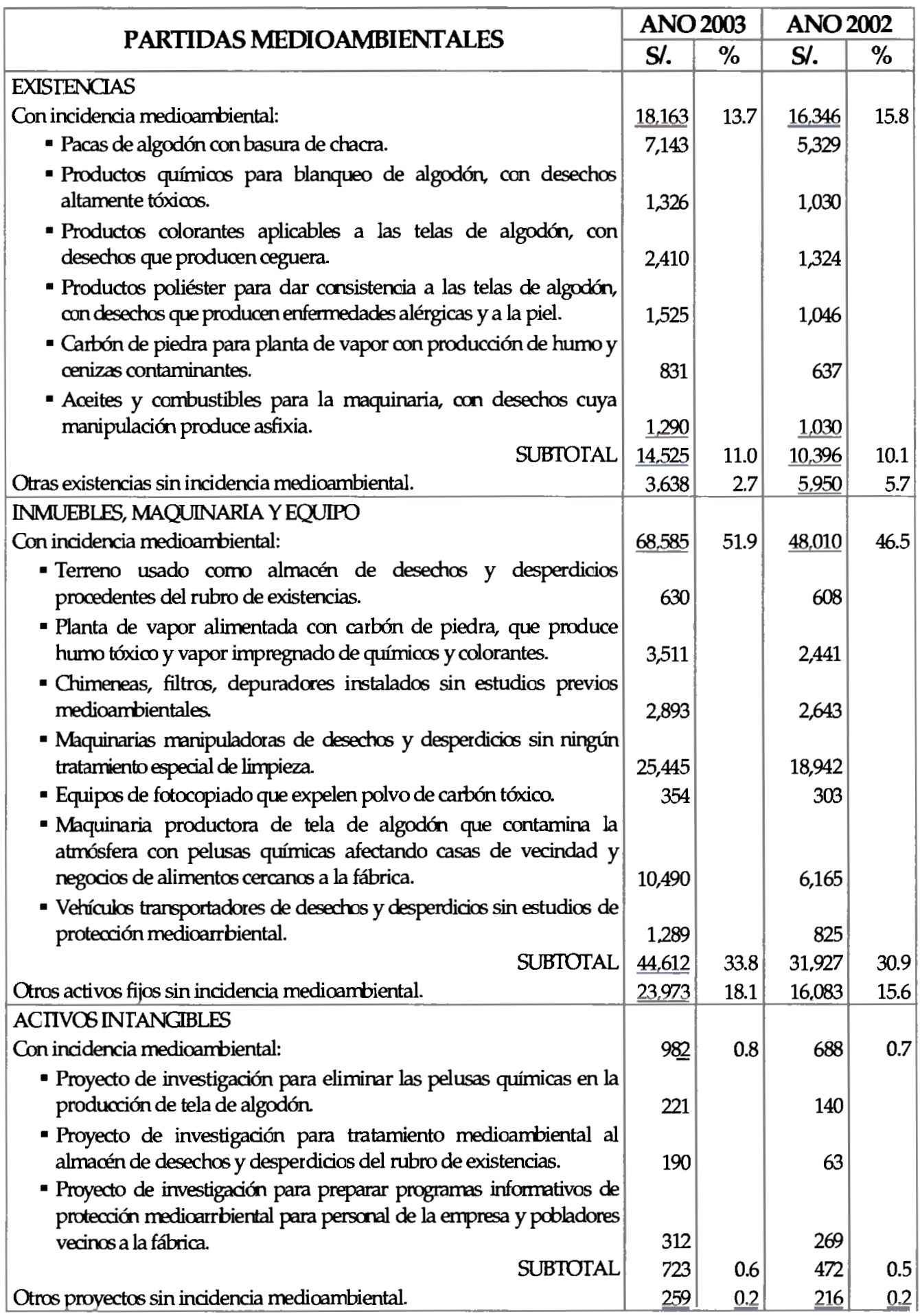




\section{ALGODONERA PERÚ S.A. PASIVO-UBICACIÓN DE RUBROS CON PARTIDAS MEDIOAMBIENTALES \\ Al 31 de diciembre del 2003 \\ (Expresado en miles de nuevos soles)}

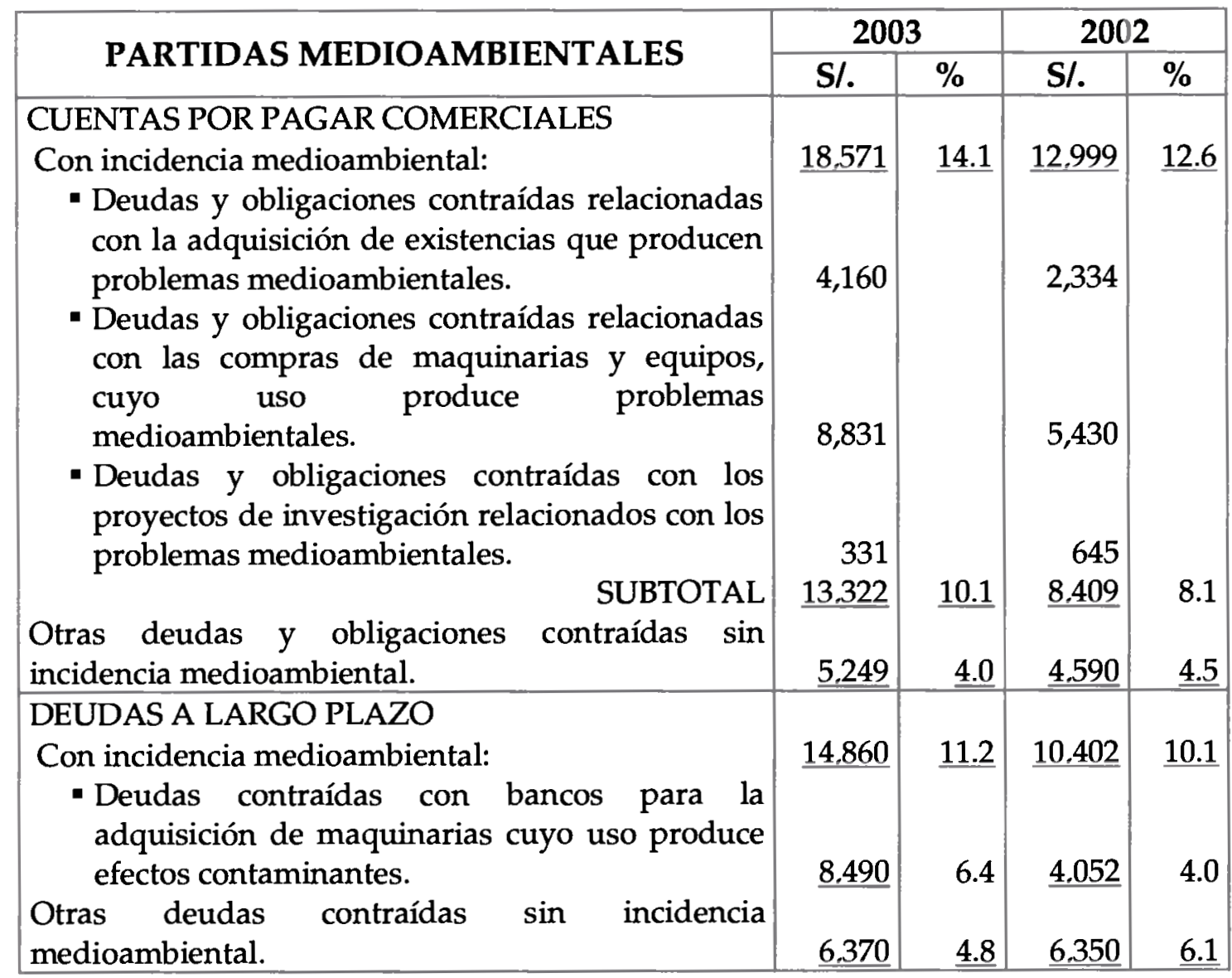

2. ESTADO DE GANANCIAS $Y$ PÉRDIDAS CON INFORMACIÓN PORCENTUAL

Al 31 de diciembre del 2003

(Expresado en miles de nuevos soles)

\section{ANEXO N. ${ }^{\circ} 2$}

Se presenta el Estado de Ganancias y Pérdidas de la empresa Algodonera Perú S.A., al 31 de diciembre del 2003, con información económica porcentual comparativa 2003-2002, en miles de nuevos soles.

Los rubros del Estado de Ganancias y Pérdidas contienen partidas con incidencia medioambiental, que es preciso identificar para conocer el comportamiento de la empresa en materia de medio ambiente. 
COSTO DE VENTAS

UBICACIÓN DE RUBROS CON PARTIDAS MEDIOAMBIENTALES

Al 31 de diciembre del 2003

(Expresado en miles de nuevos soles)

\section{SUBANEXO 2.1.}

En el Estado de Ganancias y Pérdidas mostrado en el ANEXO N. ${ }^{\circ} 2$, se han ubicado varios rubros con información medioambiental. En el SUBANEXO N. 2.1 se identifican las partidas con incidencia medioambiental del rubro de "Costo de Ventas", en los componentes de "Materia Prima" y "Gastos Indirectos de Fabricación", destacándose los montos y los porcentajes en función al $100 \%$ del rubro de «Ventas Netas», como sigue:

\begin{tabular}{|l|c|c|c|c|}
\cline { 2 - 5 } \multicolumn{1}{c|}{} & \multicolumn{4}{c|}{$\begin{array}{c}\text { Con Incidencia } \\
\text { Medioambiental }\end{array}$} \\
\cline { 2 - 5 } \multicolumn{1}{c|}{} & \multicolumn{2}{c|}{2003} & \multicolumn{2}{c|}{2002} \\
\hline $\begin{array}{c}\text { COSTOS } \\
\text { DE VENTA }\end{array}$ & S/. & $\%$ & S/. & $\%$ \\
\hline $\begin{array}{c}\text { - Materia } \\
\text { Prima }\end{array}$ & 4,383 & 10.3 & 4,433 & 15.0 \\
\hline $\begin{array}{c}\text { - Gastos } \\
\text { Indirectos de } \\
\text { Fabricación }\end{array}$ & 6,484 & 15.3 & 3,609 & 12.2 \\
\hline
\end{tabular}

Comentario: La información de las partidas con incidencia medioambiental en los componentes mencionados del rubro de "Costo de Ventas" permite evaluar la problemática que se presenta en el costo de producción de la empresa y su traslado al Estado de Ganancias y Pérdidas.
GASTOS DE VENTA Y OTROS INGRESOS

UBICACIÓN DE RUBROS CON PARTIDAS MEDIOAMBIENTALES

Al 31 de diciembre del 2003

(Expresado en miles de nuevos soles)

\section{SUBANEXO 2.2}

También se han ubicado en el Estado de Ganancias y Pérdidas, que se muestra en el ANEXO N..$^{\circ}$, dos rubros con información medioambiental, cuyo análisis se presenta en el SUBANEXO N. ${ }^{\circ} 2.2$, destacándose los montos y porcentajes en función al $100 \%$ del rubro de "Ventas Netas». El primer rubro es "Gastos de Ventas" y el segundo rubro es "Otros Ingresos". En ambos se identifican claramente las partidas con incidencia medioambiental, como sigue:

\begin{tabular}{|l|c|c|c|c|}
\cline { 2 - 5 } \multicolumn{1}{c|}{} & \multicolumn{4}{c|}{$\begin{array}{c}\text { Con Incidencia } \\
\text { Medioambiental }\end{array}$} \\
\cline { 2 - 5 } & \multicolumn{2}{c|}{2003} & \multicolumn{2}{c|}{2002} \\
\cline { 2 - 5 } & S/. & $\%$ & S/. & $\%$ \\
\hline $\begin{array}{l}\text { GASTOS DE } \\
\text { VENTAS }\end{array}$ & 1,469 & 3.5 & 819 & 2.8 \\
\hline $\begin{array}{l}\text { OTROS } \\
\text { INGRESOS }\end{array}$ & 1,480 & 3.5 & 1,077 & 3.6 \\
\hline
\end{tabular}

Comentario: Las partidas medioambientales también se ubican en diversos rubros de ingresos y gastos del Estado de Ganancias y Pérdidas, que es preciso informar. 


\section{ALGODONERA PERÚ S.A. \\ ESTADO DE GANANCIAS Y PÉRDIDAS CON INFORMACIÓN PORCENTUAL}

Al 31 de diciembre del 2003

(Expresado en miles de nuevos soles)

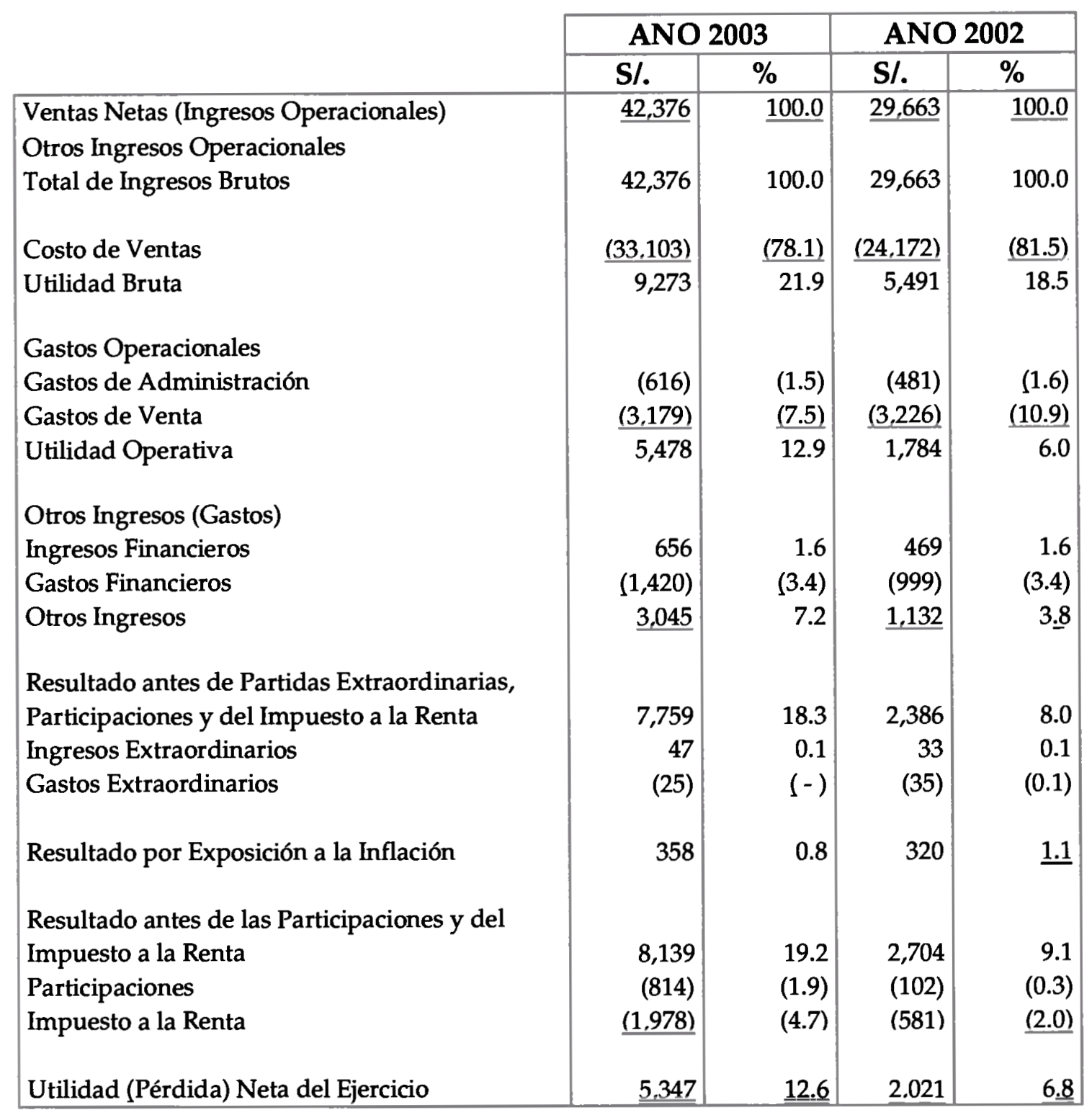




\section{ALGODONERA PERÚ S.A. \\ COSTO DE VENTAS \\ UBICACIÓN DE RUBROS CON PARTIDAS MEDIOAMBIENTALES \\ Al 31 de diciembre del 2003 \\ (Expresado en miles de nuevos soles)}

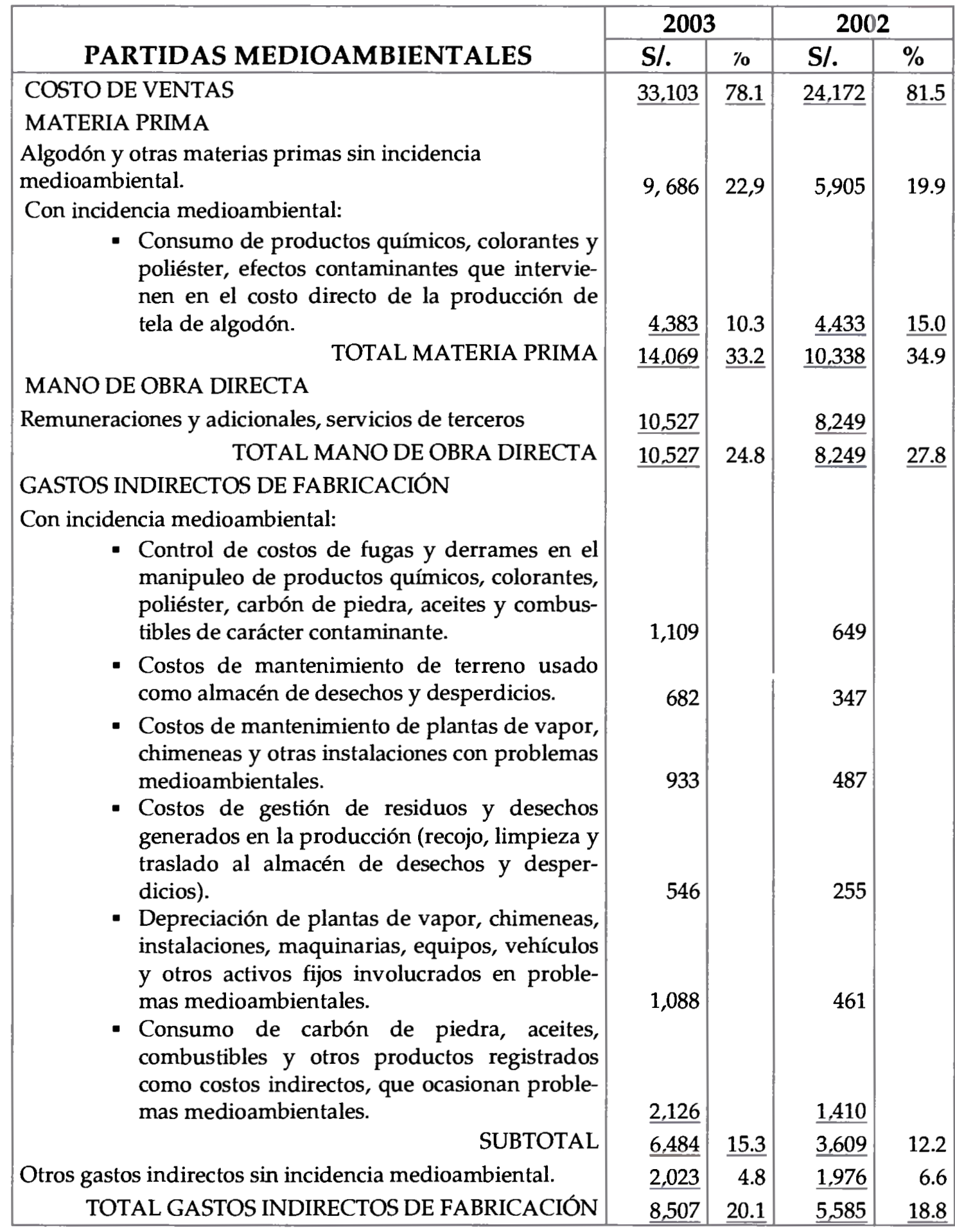


SUBANEXO 2.2

\section{ALGODONERA PERÚ S.A. GASTOS DE VENTAS Y OTROS INGRESOS UBICACIÓN DE RUBROS CON PARTIDAS MEDIOAMBIENTALES \\ Al 31 de diciembre del 2003 \\ (Expresado en miles de nuevos soles)}

\begin{tabular}{|c|c|c|c|c|}
\hline \multirow{2}{*}{ PARTIDAS MEDIOAMBIENTALES } & \multicolumn{2}{|c|}{2003} & \multicolumn{2}{|c|}{2002} \\
\hline & $\mathbf{S} /$. & $\%$ & S/. & $\%$ \\
\hline GASTOS DE VENTA & 3,179 & 7.5 & $\underline{3,226}$ & 10.9 \\
\hline Con incidencia medioambiental: & & & & \\
\hline $\begin{array}{l}\text { - Seguros medioambientales, autorizaciones, licencias, permi- } \\
\text { sos y desechos de uso de tecnología medioambiental. }\end{array}$ & 480 & & 331 & \\
\hline - Manipulación de envases de tela de algodón para la venta. & 60 & & 10 & \\
\hline - Gastos de información y formación medioambiental. & 109 & & 69 & \\
\hline $\begin{array}{l}\text { - Gastos por tratamientos medioambientales a las áreas alre- } \\
\text { dedor de la fábrica. }\end{array}$ & 681 & & 347 & \\
\hline $\begin{array}{l}\text { - Evaluaciones de impacto medioambiental que afectan las } \\
\text { ventas. }\end{array}$ & 139 & & 62 & \\
\hline SUBTOTAL & $\underline{1,469}$ & 3.5 & 819 & 2.8 \\
\hline Otros gastos de venta sin incidencia medioambiental. & 1,710 & 4.0 & 2,407 & 8.1 \\
\hline OTROS INGRESOS & $\underline{3,045}$ & 7.2 & $\underline{1,132}$ & 3.8 \\
\hline Con incidencia medioambiental: & & & & \\
\hline - Ingresos por ventas de desechos y desperdicios de algodón. & 810 & & 602 & \\
\hline $\begin{array}{l}\text { - Ingresos por ventas de envases que contenían productos } \\
\text { químicos, colorantes y otros clasificados como contaminantes. } \\
\text { - Ingresos por ventas de ceniza y carbón de piedra desme- } \\
\text { nuzado, considerados como excedentes altamente contami- } \\
\text { nantes. }\end{array}$ & 449 & & 333 & \\
\hline SUBTOTAL & $\underline{1,480}$ & 3.5 & $\underline{1,077}$ & 3.6 \\
\hline Otros ingresos sin incidencia medioambiental. & 1,565 & 3.7 & 55 & 0.2 \\
\hline
\end{tabular}

\section{CONCLUSIONES}

1. Se está demostrando de manera práctica que el Balance General y el Estado de Ganancias y Pérdidas contienen partidas con incidencia medioambiental y que es preciso extraerlas para otorgar importante información a la dirección de la empresa.
2. Por lo tanto, el Contador de la empresa está en la obligación profesional de entrenar a su personal en ubicar aquellas operaciones financieras y económicas que contienen partidas medioambientales y codificarlas, si es posible, en subcuentas que facilitan su inmediata información a la dirección de la empresa, si la gravedad de la misma así lo amerita; 
cuidando asimismo que tales partidas medioambientales sean informadas periódicamente en subanexos como los que se muestran en este artículo, para realizar mayores estudios ecológicos y búsqueda de soluciones en beneficio de mejorar el medio ambiente que rodea a la empresa y en la aplicación de los procesos de la Contabilidad Social para otorgar protección a los trabajadores y núcleos de viviendas cercanas a la empresa, en los que habitan personas, muchas veces con escasos recursos económicos para curarse de enfermedades producidas por efectos contaminantes esparcidos por las maquinas o desechos de la producción.

Es importantes también que las mismas universidades que forman Contadores Públicos y los Colegios de Contadores Públicos de todo el Perú implementen cursos y seminarios sobre Contabilidad Medioambiental y se enseñen las técnicas necesarias para que el Balance General y el Estado de Ganancias y Pérdidas otorguen información medioambiental, tal como se propone en el presente artículo. 